Instituto Internacional de Investigación y Desarrollo Tecnológico Educativo INDTEC, C.A.

DOI: https://doi.org/10.29394/scientific.issn.2542-2987.2017.0.0.5.82-94

OAI-PMH: http://www.indteca.com/ojs/index.php/Revista Scientific/oai

\title{
Huerto Escolar como Estrategia Pedagógica para el Fortalecimiento de los Valores Ambientales
}

\author{
Autor: Daniel Enrique Palma Ávila \\ Universidad Pedagógica Experimental Libertador, UPEL \\ danielpalmaavila@hotmail.com \\ Barinas, Venezuela
}

\section{Resumen}

El presente estudio tiene como objetivo general implementar el huerto escolar agroecológico como estrategia pedagógica para el fortalecimiento de los valores ambientales en los niños y niñas de la Escuela Básica Nacional "Adolfo Moreno" de la Parroquia Barinitas, Municipio Bolívar del Estado Barinas. El mismo, se desarrolló bajo el paradigma de investigación cualitativo y en el tipo investigación fue la investigación acción participativa contentiva de cuatro (4) fases (Diagnostico, Planificación, Ejecución y Evaluación), la investigación fue apoyada en un diseño de campo de carácter descriptivo. Asimismo, las técnicas utilizadas para recabar la información fue la observación participante y la entrevista, como instrumento se aplicó un guion de entrevista el cual fue respondido por tres (3) informantes claves constituido por docentes perteneciente a la institución educativa quienes suministraron la información necesaria para la descripción, interpretación y elaboración de estrategias dirigidas al logro de los objetivos planteados. La técnica de análisis de la información se llevó a cabo a través del proceso de triangulación para reducirla y así expresarla, describirla e interpretarla. Los resultados arrojaron la necesidad de la aplicación de un plan que permita la utilización del huerto escolar como estrategia pedagógica para el fortalecimiento de los valores ambientales en la institución educativa en estudio.

Palabras claves: huerto escolar; estrategias pedagógicas; valores ambientales. 


\title{
School Board as a Pedagogic Strategy for the Strengthening of Environmental Values
}

\begin{abstract}
The present study has as general objective to implement the agroecological school orchard as a pedagogical strategy for the strengthening of the environmental values in the children of the Adolfo Moreno National School of the Barinitas Parish, Bolivar Municipality of the Barinas State. The same was developed under the paradigm of qualitative research and in the research type was the participatory action research contentive of four (4) phases (Diagnostics, Design, Execution and Evaluation), the research was supported in a descriptive field design. Also, the techniques used to gather information were participant observation and interview, as an instrument was applied an interview script which was answered by three (3) key informants made up of teachers belonging to the educational institution who will provide the necessary information to the description, interpretation and elaboration of strategies directed to the achievement of the stated objectives. The technique of information analysis will be carried out through the process of triangulation to reduce it and thus express it, describe it and interpret it. The results showed the need for the implementation of a plan that allows the use of the school garden as a pedagogical strategy that allows the strengthening of environmental values.
\end{abstract}

Keywords: school garden; pedagogical strategies; environmental values.

Date Received: 24-11-2016

Date Acceptance: 23-01-2017 


\section{Introducción}

La educación en el mundo actual vive cambios significativos con planes y metas a lograr, donde, el niño, la niña y los adolescentes están inmersos. La implantación del sistema educativo ajustado a las teorías de la globalización mundial, darán como resultados la formación integral plena de los formando, bajo ciertas normas, principios que fundamente la base primordial para el desarrollo de su personalidad, del intelecto, la destreza motora, logrando una formación de calidad con valores ambientalista, de allí también el educador buscara las herramientas, las estrategias que faciliten el proceso de construcción del aprendizaje de los niños, las niñas y los adolescentes.

Por tanto, los centros de educación en todos los niveles, deben proporcionar al niño, niña y al adolescente un cúmulo de aprendizajes significativos entre los que destaca el amor por la naturaleza, conservación del ambiente, por lo que es necesario, que el docente incluya en la planificación actividades y los valores referidos a la educación ambiental, donde involucren de manera activa a los padres, madres, representantes o responsables, fomentando en ellos el deseo de conservar los recursos naturales que se encuentran en la institución. En tal sentido, Bronfenbrenner (2008: 40) destaca: "la influencia directa que tiene la educación y el entorno ambiental sobre el individuo en desarrollo, y específicamente resalta la interacción entre ambos (individuo - escuela - contexto social), como proceso generador de cambios". Esa estrecha relación que tiene la educación con la conservación ambiental depende en gran parte de la estrategia que el docente aplique desde la institución con los recursos naturales existentes en su medio, motivando a la colectividad, en primer orden a los educandos, creando en ellos conocimientos, habilidades, destrezas, valores indispensables para la protección del ambiente asegurando de esta forma el progreso de las futuras generaciones. 
Al respecto, lo planteado es evidenciado en la población educativa de la Escuela Básica Nacional "Adolfo Moreno", parroquia Barinitas, municipio Bolívar del Estado Barinas, que contando con suficiente espacio natural, no se le dé un aprovechamiento positivo para la producción agrícola ecológica o agroecológica en estas áreas, y de esta manera fomentar valores ambientales, para la producción. De igual modo, los estudiantes no cuentan con la orientación respectiva elemental sobre la importancia que tienen los huertos escolares en el desarrollo endógeno y alimentación sustentable de las comunidades.

Por consiguiente, se hace necesario la construcción de un huerto escolar agroecológico para que los niños, niñas y adolescentes aprendan sobre el ciclo biológico, el cultivo de las plantas para diversos fines, reconocimientos de semillas, construcción de semilleros, tipos de abono, riego, manejo integrado de plagas y enfermedades de los cultivos y así fomentar los valores ambientales.

Sobre la base de las consideraciones anteriores es pertinente llevar un proceso de investigación que nos permita plantear las siguientes interrogantes:

¿Cuál sería la necesidad de construcción de un huerto escolar agroecológico como estrategia pedagógica en la Escuela Adolfo Moreno de la Parroquia Barinitas, Municipio Bolívar del Estado Barinas?

¿Cómo sería el plan educativo para la construcción de un huerto escolar agroecológico como estrategia pedagógica en la Escuela Adolfo Moreno de la Parroquia Barinitas, Municipio Bolívar del Estado Barinas?

¿Cómo será la ejecución del plan para la construcción de un huerto escolar agroecológico como estrategia pedagógica para el fortalecimiento de valores ambientales en la Escuela Adolfo Moreno de la Parroquia Barinitas, Municipio Bolívar del Estado Barinas? 
¿Cuál sería el impacto del huerto escolar agroecológico como estrategia pedagógica para el fortalecimiento de valores ambientales en la Escuela Adolfo Moreno de la Parroquia Barinitas, Municipio Bolívar del Estado Barinas?

\section{Contexto Teórico.}

\subsection{Huerto Escolar}

De acuerdo con lo señalado por el Ministerio del poder Popular para la Educación (2010: 34), el huerto escolar es "un área de cultivo de hortalizas, granos, cereales y frutas entre otros, dentro de un espacio escolar o lugar próximo a la escuela". Se constituyen en gran utilidad desde la experiencia educativa, esta idea está plasmada en las orientaciones educativas para los niveles y modalidades del subsistema de educación Básica entre sus planes, programas y proyectos, específicamente en el proyecto.

\subsection{Organización del Huerto Escolar:}

Según, Rincón (2006), propone que el primer paso de organización de la huerta es: "saber lo que vamos a sembrar, hacer una lista, preparar el terreno, teniendo en cuenta la situación geográfica del huerto; así como las condiciones del clima" (pág. 15). Para ello propone tener en cuenta:

\subsubsection{El Viento}

Ubicar el huerto en una zona donde la dirección del viento no afecte el sembradío, por cuanto hay plantas que no toleran la fuerza del viento, tales como los frutales, las coles y otras de mayor tamaño. Por lo tanto, si el terreno seleccionado, está muy expuesto al viento es conveniente colocar algún tipo de defensa para lo que el autor sugiere una valla con huecos y no un muro, o en su defecto tener que sembrar arbustos a su alrededor. 


\subsubsection{El Agua}

Es conveniente, que el semillero y en el huerto se disponga de una toma de agua, para ayudar a las semillas a fijar sus raíces, una vez que éstas estén firmes, el riego regular ya no están vital, excepto en climas muy secos.

\subsubsection{Labrar el Terreno}

El método tradicional de labrar un terreno que se va a preparar para la huerta, es por medio de la pala o pico. Al desarraigar por primera vez, hay que trabajar el suelo con intensidad.

\subsection{Manejo agroecológico del Huerto}

La agricultura del futuro debe ser sostenible como altamente productiva si se desea producir alimento para una creciente población humana. Al respecto, Delgado y Marín (2009) señalan que:

el manejo agroecológico del huerto escolar implica aprovechar al máximo las bondades de la naturaleza y las mejores prácticas para la conservación de los recursos naturales elementales de la producción de alimentos, sin la utilización de abonos químicos, herbicidas y plaguicidas, labranza intensiva, entre otras prácticas de la agricultura moderna, que esfuerza a la naturaleza a producir más allá de unos límites que permitirían mantener un mínimo equilibrio biológico y ecológico del entorno. (pág. 43).

En efecto, el manejo ecológico de loa huertos escolares tiene como causa fundamental el aprovechamiento de los beneficios que nos da la madre naturaleza; asimismo, permite la producción de alimentos mediante un uso sustentable y sostenible de los recursos naturales.

\section{Metodología}

El presente estudio se enmarcó bajo el paradigma de investigación cualitativa puesto que se orienta hacia el estudio de problemas relacionados 
con la experiencia humana individual y colectiva; fenómenos sobre los que se conoce poco y se aspira comprender en su contexto natural. La misma, tiene su carácter flexible y emergente, que implica tomar decisiones en el contexto durante el proceso. En esta investigación, se involucra de manera preocupante en los acontecimientos y centra su indagación en los contextos naturales, o tomados tal y como se encuentran, donde los seres humanos se implican e interesan, evalúan y experimentan directamente. Con esta intención se utiliza el método cualitativo como herramienta que permita estudiar, conocer o comprender individuos y grupos, tanto en el entorno social, personal como escolar.

De la misma manera, el tipo de investigación que se emplea en este estudio, es la Investigación Acción Participativa la cual, busca una forma de investigación social y una modalidad de la investigación interactiva, la cual se caracteriza entre otras cosas por el hecho de que la acción, más que un producto de interés particular del investigador, esta surge como necesidad de un grupo o comunidad y es llevada a cabo por los involucrados en la acción a modificar, de modo que el investigador actué como facilitador del proceso.

Esto indica, que la investigación acción conlleva a la modificación de una realidad, como parte del proceso investigativo donde se involucra al grupo en la generación de su propio conocimiento y en la sistematización de su propia experiencia.

En tal sentido, Elliot (1990), por su parte, considera la investigación-acción como:

El estudio de una situación social para intentar mejorar la calidad de la acción en la misma. Su objetivo consiste en proporcionar elementos que sirvan para facilitar el juicio práctico en situaciones concretas y la validez de las teorías e hipótesis que genera no depende tanto de pruebas 'científicas' de verdad, sino de su utilidad para ayudar a las personas a actuar de modo más inteligente y acertado [...] En la investigación acción, las 'teorías' no se validan de forma 
independiente para aplicarlas luego a la práctica, sino a través de la práctica (pág. 88).

El esquema metodológico propuesto por este autor, está constituido por las siguientes fases:

Fase I. Diagnóstico: origina el primer momento de búsqueda, de indagación, acompañado de un gran deseo de comprender el por qué ocurren las cosas. La reflexión en el momento del diagnóstico permite conocer los problemas o situaciones que afectan a un grupo o comunidad. Con la aplicación de la técnica de entrevista y la observación participante y con los instrumentos de registro audiovisual y el guion de entrevista. Por ello una vez identificado el significado del problema, y, formulado el enunciado del mismo, se debe proceder a buscar alternativas de solución para solventar la problemática existente.

Es por tanto, que en el presente estudio, el diagnóstico será participativo porque en el mismo estará conformado por los docentes de la Unidad Educativa "Adolfo Moreno" de la Parroquia Barinitas del Municipio Bolívar.

Fase II. Planificación: El presente estudio, el plan de acción partirá de las iniciativas y consensos logrados durante las interacciones con los involucrados en la investigación, permitiéndole dar vías de solución a las problemáticas presente en la comunidad de estudio. De la misma manera, corresponde en este aparte el momento de definir las estrategias que mejor se adapten a las necesidades den estrategias pedagógicas utilizando el huerto escolar para fortalecer los valores ambientales.

Fase III Ejecución: En este caso, la ejecución del plan de acción, obtendrá significativas experiencias tanto para el investigador como por los investigados, si se toma en cuenta los resultados de la evaluación realizada durante el desarrollo de cada una de las actividades previstas para el alcance de los objetivos. En este sentido, partiendo de las acciones realizadas en la 
fase anterior que relacionadas con la planificación de las estrategias que conformarán el plan se procederá a la aplicación de las misma, de manera tal que este permita generar acciones que resuelvan la problemática de perdida de los valores ambientales en los educandos de la unidad educativa en estudio.

Fase IV Evaluación: En esta fase, se desarrollarán acciones destinadas a la revisión de la ejecución del plan atendiendo a criterios establecidos para determinar la efectividad e impacto que obtendrá con la aplicación de las estrategias pedagógicas para fortalecer los valores ambientales utilizando el huerto escolar como herramienta.

Igualmente, el diseño de la investigación se enmarca en un diseño de campo, la cual consiste en aproximarse a la realidad de lo que sucede en una comunidad o un contexto socio-geográfico específico. Asimismo, este diseño realiza un análisis sistemático de problemas en la realidad, con el propósito bien sea de describirlos, interpretarlos, entender su naturaleza y factores constituyentes, explicar sus causas y efectos a predecir su concurrencia, haciendo uso de métodos característicos de cualquiera de los paradigmas o enfoques de investigación conocidos o en desarrollo.

Lo planteado, especifica que el diseño de campo busca el contexto real donde están ocurriendo los hechos; por tal motivo los datos que se obtendrán en esta investigación se obtendrán de las opiniones emitidas por los sujetos pertenecientes a la Escuela Básica Nacional "Adolfo Moreno" de la Parroquia Barinitas, Municipio Bolívar del Estado Barinas.

En este orden de ideas la investigación se enmarca en un carácter descriptivo, porque la mismo busco ampliar a través de la interpretación de la realidad estudiada, esta investigación de tipo descriptiva trabaja sobre realidades de hechos, y su característica fundamental es la de presentar una interpretación correcta. Para la investigación descriptiva, su preocupación primordial radica en descubrir algunas características fundamentales de 
conjuntos homogéneos de fenómenos, utilizando criterios sistemáticos que permitan poner de manifiesto su estructura o comportamiento. De esta forma, se pueden obtener las notas que caracterizan a la realidad estudiada.

Técnica de recolección de Información, se utilizará como técnicas la observación participante es la técnica más utilizada en la investigación cualitativa una implicación del observador en los acontecimientos o fenómenos que está observando. La implicación supone participar en la vida social y compartir actividades que realizan las personas que forman parte de la comunidad de una institución. La misma, permitirá conocer la realidad, como acción inicial, sobre el escenario de los acontecimientos de tal manera que se podrá abordar mayor información y sacar provecho de las experiencias que se manifestarán en ese momento. Igualmente, se utilizará la aplicación de una entrevista. Al respecto, la entrevista constituye el instrumento principal de recolección de información que permite la elaboración de la investigación. De esta manera, dicha entrevista, se aplicará a tres (3) docentes de la Unidad Educativa "Adolfo Moreno"; la misma se llevará a cabo dándole utilidad a un guion de entrevista manteniendo el formato de preguntas abiertas y personales con el fin de propiciar que los entrevistados expresen con sus propias palabras, la perspectiva personal sobre el tema.

A través de la interpretación de estas entrevistas se permitirá el reconocimiento de las interrelaciones con el entorno de todos los actores claves y así entrever el problema, la posible solución y la disposición al cambio, lo cual servirá de marco de referencia para establecer la planificación de la acción.

El análisis e interpretación surgirá a partir de la observación y de una entrevista aplicada a los docentes, los cuales se analizarán e interpretarán mediante el Método Comparativo Constante. Las categorías emergen del discurso, el cual es transcrito, en lo posible, de manera tal y como ocurrió, de modo que posteriormente se agrupan y codifican en subconjunto de datos 0 
frases que tuvieran afinidad entre las planteadas por dichos actores asignándolas como categorías y sub categorías. En tal sentido, la investigación consta de tres (3) categorías (Hurto Escolar, Estrategias pedagógicas y Valores Ambientales.).

En este contexto, en el análisis de los resultados se aplicará el proceso de categorización primero se revisa la información recolectada, con la actitud de conocer la realidad del fenómeno estudiado, reflexionar sobre la situación concreta para comprenderla en su total dimensión. Habida cuenta, que los intentos cognoscitivos para generar conocimientos son procesos hermenéuticos caracterizados por las interpretaciones sucesivas de los escenarios de los fenómenos sociales. Para ello, se analizan varias veces la información recopilada.

El primer análisis; se hace con el fin de tener una amplia visión de conjunto que asegure el proceso de categorización, se realizan nuevas revisiones para captar aspectos, detalles no detectados con anterioridad o, que no hayan sido valorados suficientemente lo cual, puede fortalecer su significado. Seguidamente, se resaltan las expresiones más significativas, construyendo esquemas de interpretación, donde emergen las categorías y sus propiedades 0 atributos.

\section{Consideraciones Finales}

Tomando en cuenta el proceso de investigación y considerando las necesidades diagnosticadas en la institución educativa estudiada, las conclusiones permitieron diagnosticar algunas debilidades en cuanto a la pérdida de valores ambientales. Por tanto, arrojaron la necesidad de implementar un plan que permita del huerto escolar como estrategia pedagógica para el fortalecimiento de los valores ambientales y que el mismo este dirigido a los docentes de la Unidad Educativa "Adolfo Moreno" de la Parroquia Barinitas, Municipio Bolívar del Estado Barinas. 


\section{Referencias}

Arias, F. (2004). EI Proyecto de Investigación. (4ta edición). Caracas: Editorial Epistema.

Berger, P. y Luckmann, T. (1968). La construcción social de la realidad. Argentina, Buenos Aires: Amorrortu.

Delgado y Marín (2009). Manejo Ecológico del Huerto Escolar en el Proceso de Integración Escuela-Comunidad. Universidad de los Andes. Trujillo, Venezuela.

Duarte, B. (2011). El Huerto Escolar como alternativa para preservar el medio ambiente en la Comunidad Escolar del Taller de Educación Laboral Bolivariano "San Cristóbal”. San Cristóbal, Táchira: UPEL. Recuperado de: https://es.scribd.com/document/351176576/Trabajode-Fase-Ejecucion-de-Un-Proyecto

Martínez, M. (2004). Ciencia y Arte en la Metodología Cualitativa. Trillas México.

Rojas, B. (2010). Investigación Cualitativa. Segunda edición. Caracas: FEDUPEL.

Sabino, C. (2002). El proceso de Investigación. Editorial PANAPO. Caracas. Universidad Pedagógica Experimental Libertador. Vicerrectorado de Investigación y Postgrado (2012). Cuarta edición. Manual de Trabajos de Especialización y Maestría y Tesis Doctorales. Caracas

Vásquez, M. (2011). La Integración de la estrategia Huerto Escolar y su contribución al mejoramiento del proceso de enseñanza y de aprendizaje en el currículo del nivel elemental. Río Piedras, Puerto Rico: Universidad Metropolitana, Escuela Graduada de Educación. [Documento en línea]. Recuperado de:

http://www.anagmendez.net/umet/pdf/biblioteca_tesisedu_vazquezcolo nm2011.pdf 


\section{Daniel Enrique Palma Ávila \\ e-mail: danielpalmaavila@hotmail.com}

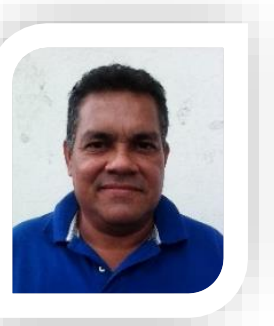

Nació en Barinas, Venezuela, el 27 de febrero de 1971, realizo sus estudios universitarios, en el Instituto Universitario de Tecnología Isaac Newton Extensión Barinas donde obtuvo el título de Técnico Superior Universitario en Educación Integral. Asimismo, le dio continuidad a sus estudios en la Universidad Cecilio Acosta donde se graduó como Licenciado en Educación Integral. En la actualidad, es cursante de la maestría en Educación Ambiente y Desarrollo de la Universidad Pedagógica Experimental Libertador núcleo académico Barinas. Igualmente, ha participado en diferentes congresos nacionales e internacionales como el congreso internacional de Innovación e investigación Educativa realizado por la Universidad Nacional Experimental de los Llanos Ezequiel Zamora.

El contenido de este manuscrito se difunde bajo una Licencia de Creative Commons ReconocimientoNoComercial-Compartirlgual 4.0 Internacional 